\title{
Deep brain stimulation of the ventralis intermedius nucleus for the treatment of essential tremor
}

\author{
Alexander G. Chartrain, BS, Ahmed J. Awad, MD, Jonathan J. Rasouli, MD, \\ Robert J. Rothrock, MD, and Brian H. Kopell, MD \\ Department of Neurosurgery, Mount Sinai Hospital, New York, New York

\begin{abstract}
A 59-year-old woman with a 30-year history of essential tremor refractory to medical therapy underwent staged deep brain stimulation of the ventralis intermedius nucleus of the thalamus (VIM). Left-sided lead placement was performed first. Once in the operating room, microelectrode recording (MER) was performed to confirm the appropriate trajectory and identify the VIM border with the ventralis caudalis nucleus. MER was repeated after repositioning $2 \mathrm{~mm}$ anteriorly to reduce the likelihood of stimulation-induced paresthesias. Physical examination prior to permanent lead placement demonstrated micro-lesion effect, suggesting optimal trajectory. After implantation of the permanent lead, physical examination showed excellent results.
\end{abstract}

The video can be found here: https://youtu.be/nn3KRdmRCZ4.

KEYWORDS deep brain stimulation; ventralis intermedius nucleus of the thalamus; essential tremor 